\title{
Lysophosphatidic acid enhances neointimal hyperplasia following vascular injury through modulating proliferation, autophagy, inflammation and oxidative stress
}

\author{
XUHUI SHEN, JIANJUN ZOU, FUYONG LI, TIANHE ZHANG and TONGQI GUO \\ Third Department of Neurosurgery, The People's Hospital of China Medical University, The \\ People's Hospital of Liaoning Province, Shenyang, Liaoning 110016, P.R. China
}

Received November 1, 2017; Accepted March 21, 2018

DOI: $10.3892 / \mathrm{mmr} .2018 .8937$

\begin{abstract}
Lysophosphatidic acid (LPA), which is one of the intermediate products of membrane phospholipid metabolism, is a bioactive phospholipid that possesses diverse activities. In the present study, the effects of LPA on neointimal formation following vascular injury were investigated. A carotid artery balloon injury model was employed in the present study, and following vascular injury, rats received an intraperitoneal injection of $1 \mathrm{mg} / \mathrm{kg}$ LPA. Subsequently, histopathological alterations were assessed by hematoxylin and eosin staining, the expression levels of proliferating cell nuclear antigen (PCNA) were detected by immunohistochemistry, apoptosis was assessed via a terminal deoxynucleotidyl transferase-mediated dUTP nick end labeling assay, and the expression levels of apoptosis-associated and autophagy-associated proteins were detected by western blotting. In addition, inflammatory and oxidative stress-associated factors were assessed by reverse transcription-quantitative polymerase chain reaction or corresponding kits. The results of the present study demonstrated that LPA enhanced vascular injury-induced neointimal hyperplasia. LPA further elevated the expression levels of PCNA
\end{abstract}

Correspondence to: Dr Xuhui Shen, Third Department of Neurosurgery, The People's Hospital of China Medical University, The People's Hospital of Liaoning Province, 33 Wenyi Road, Shenyang, Liaoning 110016, P.R. China

E-mail: shenxuhui2006@126.com

Abbreviations: Bcl-2, B-cell lymphoma-2; Bax, Bcl-2-associated X protein; DAB , 3, 3'-diaminobenzidine; HE, hematoxylin and eosin; HRP, horseradish peroxidase; IL, interleukin; LPA, lysophosphatidic acid; MDA, malondialdehyde; MPO, myeloperoxidase; PCNA, proliferating cell nuclear antigen; RT-qPCR, reverse transcription-quantitative polymerase chain reaction; SOD, superoxide dismutase; TBST, Tris-buffered saline with Tween; TNF, tumor necrosis factor; TUNEL, terminal deoxynucleotidyl transferase-mediated dUTP nick end labeling

Key words: apoptosis, autophagy, inflammation, lysophosphatidic acid, neointimal hyperplasia, oxidative stress, proliferation in the injured carotid artery tissues. LPA exhibited no effect on apoptosis in carotid artery tissues, whereas it modulated autophagy in the injured carotid artery tissues. Furthermore, LPA enhanced vascular injury-induced inflammation and oxidative stress. The present study demonstrated that LPA may enhance neointimal hyperplasia following vascular injury by modulating proliferation, autophagy, inflammation and oxidative stress, but not apoptosis. Furthermore LPA may contribute to the pathology of atherosclerosis and may be considered a promising therapeutic target for the treatment of atherosclerosis.

\section{Introduction}

Atherosclerosis is a complex arterial disease characterized by lipid accumulation, inflammation and matrix remodeling in the arterial walls. Low-density lipoprotein retention and endothelial cell activation initiate the formation of atherosclerotic lesions in the arterial intima (1). In the early stages of lesion formation, monocytes are recruited to the vascular walls and subsequently engulf lipids; after which, monocytes are transformed to foam cells (2). Neointimal formation is an early step in the development of atherosclerotic plaques (3). During atherosclerosis, vascular smooth muscle cells proliferate and migrate to the intima, contributing to the thickening of vascular intima associated with the pathogenesis of atherosclerosis $(4,5)$. In the advanced stage of lesion formation, the accumulation of lipids, extracellular matrix and vascular smooth muscle cells form fibroatheroma in the intima (2). The narrowing of vessels influences oxygen supply, thus resulting in the ischemia of tissues, including the myocardium and brain. Atherosclerosis is one of the major causes of heart attacks and strokes, and is responsible for $>50 \%$ of all cases of mortality in developed countries (6).

Lysophosphatidic acid (LPA) is one of the intermediate products of membrane phospholipid metabolism and is a bioactive phospholipid present in almost all tissues (7). LPA has been reported to possess various activities that affect survival, development, morphological alterations and inflammation, via G-protein-coupled receptors $(7,8)$. LPA is also involved in numerous diseases, including neurological disorders, cardiovascular diseases, fibrosis, tumors and inflammation $(8,9)$. 
Furthermore, LPA is associated with various vessel wall cell activities, and contributes to vasculogenesis, angiogenesis and vascular remodeling (7,10-12). Indirect evidence that blockade of LPA receptors reduces neointimal hyperplasia has demonstrated that LPA may be involved in neointimal formation following vascular injury and LPA receptor blockade relieves atherosclerotic development $(13,14)$; however, the effects of LPA on neointimal formation remain unclear.

In the present study, the effects of LPA on neointimal formation following vascular injury were investigated. The findings indicated that LPA may contribute to the pathology of atherosclerosis, and may be considered a promising therapeutic target for the treatment of atherosclerosis.

\section{Materials and methods}

Animal experimental protocol. Healthy Sprague Dawley rats ( $\mathrm{n}=36$; male; age, 8 weeks; weight, 240-260 g) were obtained from Liaoning Changsheng Biotechnology Co., Ltd. (Benxi, China) and were housed in a standard environment with controlled temperature $\left(21-23^{\circ} \mathrm{C}\right)$, humidity $(45-55 \%)$, lighting (12 h light/dark cycle) and free access to food and water. Rats in the vascular injury group and vascular injury + LPA group ( $\mathrm{n}=12$ for each group) underwent carotid artery balloon injury. Briefly, rats were anesthetized and fastened in a supine position. A midline incision was performed on the skin of the anterior neck. The left common carotid, internal and external carotid arteries were exposed via an anterior incision of the neck. A $2.0 \mathrm{~F}$ balloon catheter was introduced via the external carotid artery and advanced towards the proximal end until it reached the common carotid artery. The balloon was inflated with 2-fold atmospheric pressure to obstruct the bloodstream for $30 \mathrm{sec}$. The common carotid artery was injured by passing the inflated balloon back and forth slowly three times. Subsequently, the catheter was removed and the external carotid arteries and incision were closed. Following the surgical procedure, rats received penicillin $\left(2 \times 10^{5}\right.$ units; intramuscular injection; Harbin Motian Agricultural Technology Veterinary Drug Co., Ltd., Harbin, China) to prevent infection. Rats in the sham group received similar operations, but no balloon-induced injury. Following balloon-induced injury, rats in the vascular injury + LPA group received LPA (1 $\mathrm{mg} / \mathrm{kg}$, intraperitoneal injection; Aladdin Industrial Corporation, Shanghai, China) every 2 days for 35 days (18 injections in total). Rats in the sham $(n=12)$ and vascular injury groups received an equal amount of normal saline (intraperitoneal injection). After 35 days, the carotid artery tissues were harvested for subsequent experiments. The present study followed the Guide for the Care and Use of Laboratory Animals (15) and was approved by the Ethics Committee of The People's Hospital of China Medical University (Liaoning, China).

Hematoxylin and eosin (HE) staining. The carotid artery tissues were fixed in $4 \%$ paraformaldehyde for $24 \mathrm{~h}$ at room temperature, dehydrated in graded ethanol, cleared with xylene, then embedded in paraffin and cut into 5- $\mu \mathrm{m}$ sections. The sections were deparaffinized in xylene and rehydrated in graded ethanol series. Subsequently, the sections were stained with hematoxylin for $5 \mathrm{~min}$ and eosin for $3 \mathrm{~min}$ at room temperature. Images of carotid artery tissues in each group were obtained using light microscopy with the cellSens Entry 1.9 imaging system (Olympus Corporation, Tokyo, Japan). According to the HE staining images, the intima, tunica media and lumen areas were calculated.

Immunohistochemistry. Following deparaffinization and rehydration, the paraffin-embedded sections were maintained in citrate buffer at $100^{\circ} \mathrm{C}$ for $10 \mathrm{~min}$ for antigen retrieval. Following rinsing with $\mathrm{PBS}$, the sections were incubated with $3 \%$ hydrogen peroxide at room temperature for $15 \mathrm{~min}$ to inactivate the endogenous peroxidases. Subsequently, the sections were rinsed with PBS and incubated with normal goat serum (Beijing Solarbio Science \& Technology, Co., Ltd., Beijing, China) at room temperature for $15 \mathrm{~min}$ to block nonspecific binding sites. The sections were then incubated with a primary antibody against proliferating cell nuclear antigen (PCNA; 1:50; Santa Cruz Biotechnology, Inc., Dallas, TX, USA; cat. no. sc-25280) overnight at $4^{\circ} \mathrm{C}$. Following rinsing with PBS, the sections were incubated with corresponding biotin-labeled secondary antibody (1:200; Beyotime Institute of Biotechnology, Haimen, China; cat. nos. A0286 and $\mathrm{A} 0216$ ) for $30 \mathrm{~min}$ at $37^{\circ} \mathrm{C}$. The sections were then rinsed with PBS and incubated with horseradish peroxidase (HRP)-labeled avidin (Beyotime Institute of Biotechnology) at $37^{\circ} \mathrm{C}$ for $30 \mathrm{~min}$. After further rinsing, the sections were visualized with a 3,3'-diaminobenzidine (DAB) kit (Beijing Solarbio Science \& Technology, Co., Ltd.) and counterstained with hematoxylin (Beijing Solarbio Science \& Technology, Co., Ltd.) at room temperature for $3 \mathrm{~min}$. Images of each group were obtained using a light microscope with cellSens Entry 1.9 imaging system (Olympus Corporation). The percentage of PCNA-positive cells in neointima was recorded.

Terminal deoxynucleotidyl transferase-mediated dUTP nick end labeling (TUNEL) assay. The paraffin-embedded sections were deparaffinized, rehydrated and subjected to a TUNEL assay using an In situ Cell Death Detection kit (Roche Diagnostics GmbH, Mannheim, Germany) according to the manufacturer's protocols. Briefly, the sections were permeabilized with $0.1 \%$ Triton X-100 for 8 min and blocked with $3 \%$ hydrogen peroxide for $10 \mathrm{~min}$. Subsequently, the sections were incubated with a mixture of enzyme solution and label solution (1:9) from the kit, at $37^{\circ} \mathrm{C}$ for $60 \mathrm{~min}$ in the dark. Following rinsing with $\mathrm{PBS}$, the sections were incubated with Converter-POD at $37^{\circ} \mathrm{C}$ for $30 \mathrm{~min}$. The sections were visualized with a DAB kit and counterstained with hematoxylin as aforementioned. Images were obtained using a light microscope with cellSens Entry 1.9 imaging system at x400 magnification. The percentage of TUNEL-positive cells in neointima was recorded.

Reverse transcription-quantitative polymerase chain reaction $(R T-q P C R)$. Total RNA was extracted from carotid artery tissues using a RNApure High-purity Total RNA Rapid Extraction kit (BioTeke Corporation, Beijing, China) and reverse transcribed to cDNA. The reaction system included $1 \mu \mathrm{l}$ oligo(dT)15, $1 \mu \mathrm{l}$ random primer, $2 \mu \mathrm{l}$ dNTPs, $10.5 \mu \mathrm{lddH_{2 }}$ O, $4 \mu \mathrm{l} 5 \mathrm{x}$ Buffer, $0.5 \mu \mathrm{l}$ RNasin and $1 \mu \mathrm{l}$ moloney 
murine leukemia virus reverse transcriptase (BioTeke Corporation). The thermocycling conditions were: $25^{\circ} \mathrm{C}$ for $10 \mathrm{~min}$, followed by $42^{\circ} \mathrm{C}$ for $50 \mathrm{~min}$. Subsequently, the mRNA expression levels of tumor necrosis factor (TNF)- $\alpha$, interleukin (IL)-10 and IL-1 $\beta$ were measured via qPCR with cDNA as templates. The primer sequences were as follows: TNF- $\alpha$, forward 5'-TGGCGTGTTCATCCGTTCT-3', reverse 5'-CCACTACTTCAGCGTCTCGT-3'; IL-10, forward 5'-CCA GTCAGCCAGACCCACAT-3', reverse 5'-GCATCACTT CTACCAGGTAAAAC-3'; IL-1 $\beta$, forward 5'-GGGATGATG ACGACCTGC-3', reverse 5'-ACTTGTTGGCTTATGTTC TG-3'; and $\beta$-actin, forward 5'-GGAGATTACTGCCCTGGC TCCTAGC-3' and reverse 5'-GGCCGGACTCATCGTACT CCTGCTT-3'. RT-qPCR was performed on an Exicycler ${ }^{\mathrm{TM}}$ 96 real-time PCR instrument (Bioneer Corporation, Daejeon, Korea). The thermocycling conditions were as follows: Initial denaturation at $94^{\circ} \mathrm{C}$ for $10 \mathrm{~min}$; followed by 40 cycles of $94^{\circ} \mathrm{C}$ for $10 \mathrm{sec}, 60^{\circ} \mathrm{C}$ for $20 \mathrm{sec}$ and $72^{\circ} \mathrm{C}$ for $30 \mathrm{sec}$; and final extension at $72^{\circ} \mathrm{C}$ for $150 \mathrm{sec}$. The relative mRNA expression levels were normalized to $\beta$-actin and calculated using the $2^{-\Delta \Delta \mathrm{Cq}}$ method (16).

Western blot analysis. Proteins from the samples in each group were extracted using radioimmunoprecipitation assay lysis buffer (Beyotime Institute of Biotechnology) with $1 \%$ phenylmethanesulfonyl fluoride (Beyotime Institute of Biotechnology). Following determination of the protein concentration via a bichinconinic acid (BCA) protein assay kit (Beyotime Institute of Biotechnology), the proteins $(30 \mu \mathrm{g} /$ lane) were separated by $13 \%$ SDS-PAGE. Following electrophoresis, the proteins were transferred onto polyvinylidene fluoride membranes (Merck KGaA, Darmstadt, Germany). The membranes were blocked with $5 \%$ skim milk for $1 \mathrm{~h}$ at room temperature and incubated with primary antibodies against B-cell lymphoma-2 (Bcl-2; 1:400, Wuhan Boster Biological Technology, Ltd., Wuhan, China; cat. no. BA0412), Bcl-2-associated X protein (Bax; 1:500, Sangon Biotech Co., Ltd., Shanghai, China; cat. no. D120073), caspase-3 (1:1,000, Abcam, Cambridge, UK; cat. no. ab2302), microtubule-associated protein 1A/1B light chain 3 (LC3 II/I; 1:500, Cell Signaling Technology, Inc., Danvers, MA, USA; cat. no. 2775), p62 (1:500, Cell Signaling Technology, Inc.; cat. no. 5114) or $\beta$-actin $(1: 1,000$, Santa Cruz Biotechnology, Inc.; cat. no. sc-47778) overnight at $4^{\circ} \mathrm{C}$. Subsequently, the membranes were rinsed with Tris-buffered saline with $0.15 \%$ Tween-20 (TBST) and incubated with HRP-labeled secondary antibodies (1:5,000; Beyotime Institute of Biotechnology; cat. nos. A0208 and A0216) at $37^{\circ} \mathrm{C}$ for $45 \mathrm{~min}$. The membranes were rinsed with TBST and visualized with a BeyoECL Plus enhanced chemiluminescence reagent (Beyotime Institute of Biotechnology). The optical densities of targeted bands were analyzed using Gel-Pro-Analyzer software 4 (Media Cybernetics, Rockville, MD, USA).

Measurement of malondialdehyde (MDA), superoxide dismutase (SOD) and myeloperoxidase (MPO) levels. The carotid artery tissues were homogenized in PBS, and underwent repeated freezing and thawing three times in liquid nitrogen. Following centrifugation at $10,005 \mathrm{x} \mathrm{g}$ at $4^{\circ} \mathrm{C}$ for
$10 \mathrm{~min}$, the supernatants were collected and the protein concentrations were measured using a BCA protein assay kit. Subsequently, the levels of MDA and SOD were measured using an MDA determination kit (Nanjing Jiancheng Bioengineering Institute, Nanjing, China; cat. no. A003-1) or a SOD determination kit (WST-1 method) (Nanjing Jiancheng Bioengineering Institute; cat. no. A001-3), according to the manufacturer's protocols. The carotid artery tissues were homogenized in normal saline, and the MPO levels were measured using an MPO determination kit (Nanjing Jiancheng Bioengineering Institute; cat. no. A044), according to the manufacturer's protocols.

Statistical analysis. The results are presented as the means \pm standard deviation. Differences between groups were calculated using one-way analysis of variance followed by Bonferroni's correction in GraphPad Prism 5.0 (Graphpad Software, Inc., La Jolla, CA, USA). Experiments were repeated three times. $\mathrm{P}<0.05$ was considered to indicate a statistically significant difference.

\section{Results}

LPA enhances vascular injury-induced neointimal thickening. Following vascular injury, neointimal formation of carotid artery tissues was assessed by HE staining. As presented in Fig. 1, the carotid artery tissues in the sham group revealed a common vascular structure with almost nonvisible intima; however, following vascular injury, neointima was visible, with a thickened intima. This thickening of the intima in the injured arteries appeared to be enhanced upon LPA treatment (Fig. 1A). Lumen, intimal and tunica media areas were also analyzed. Compared with the sham group, lumen area was significantly decreased in the vascular injury group $\left(0.781 \pm 0.096 \mathrm{~mm}^{2}\right.$ vs. $0.588 \pm 0.095 \mathrm{~mm}^{2}$; Fig. 1B). The lumen area further decreased following treatment with LPA (Fig. 1B). Conversely, compared with the sham group, intimal area was significantly increased by $0.279 \pm 0.037 \mathrm{~mm}^{2}$ in the vascular injury group, and further increased in the vascular injury + LPA group (Fig. 1C). In addition, no significant alterations in tunica media area were observed across the three groups (Fig. 1D). The intima/tunica media ratio was increased by $0.394 \pm 0.069$ in the vascular injury group, and further increased in the vascular injury + LPA group (Fig. 1E). These results demonstrated that intimal thickening caused by vascular injury may be enhanced by treatment with LPA.

LPA modulates proliferation and autophagy, but not apoptosis in neointima. To further evaluate neointimal hyperplasia, the expression levels of PCNA in the carotid artery tissues were detected by immunohistochemistry. The carotid artery tissues in the vascular injury group exhibited significantly increased PCNA expression levels compared with in the sham group. In addition, the carotid artery tissues in the vascular injury + LPA group demonstrated significantly higher PCNA levels compared with in the vascular injury group (Fig. 2). These results indicated that LPA enhanced proliferation of neointima.

Apoptosis is very important in the formation of neointima (17); therefore, in the present study, the extent 
A

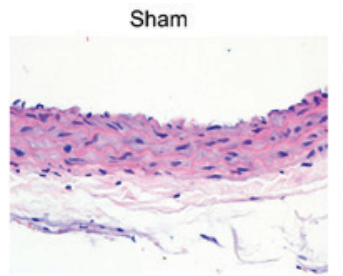

B
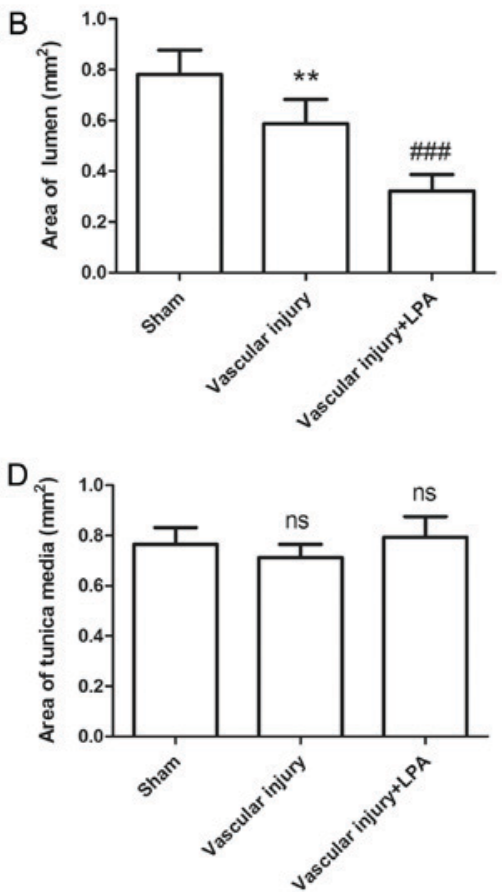

Vascular injury

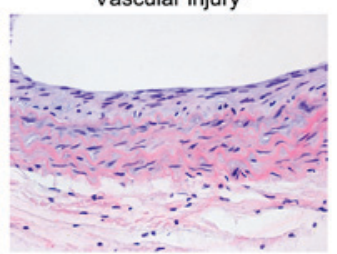

Vascular injury+LPA

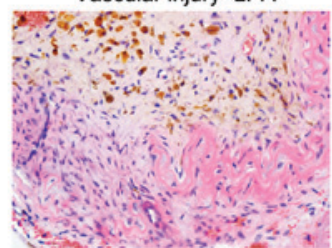

C

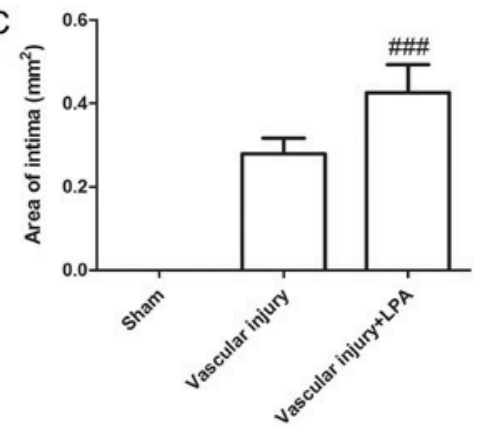

E

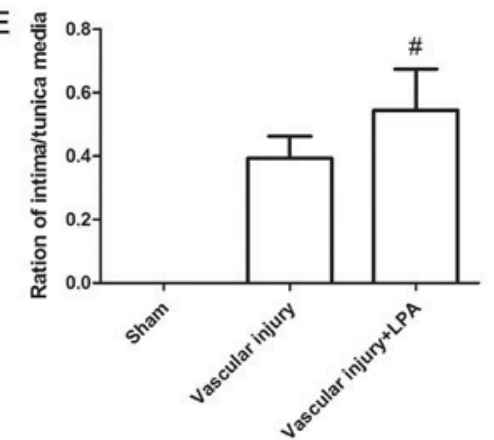

Figure 1. LPA enhances vascular injury-induced neointima formation. (A) Carotid artery tissues were collected and subjected to hematoxylin and eosin staining. Typical images are presented (magnification, $x 400$ ). (B) Lumen, (C) intimal and (D) tunica media areas, and (E) ratio of intima/tunica media in each group were calculated. Data are presented as the means \pm standard deviation ( $\mathrm{n}=6$ animals/group). ${ }^{* *} \mathrm{P}<0.01$ compared with the sham group; ${ }^{*} \mathrm{P}<0.05$ and

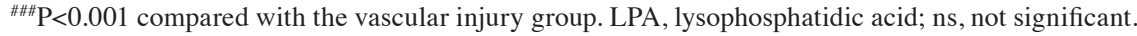

Sham

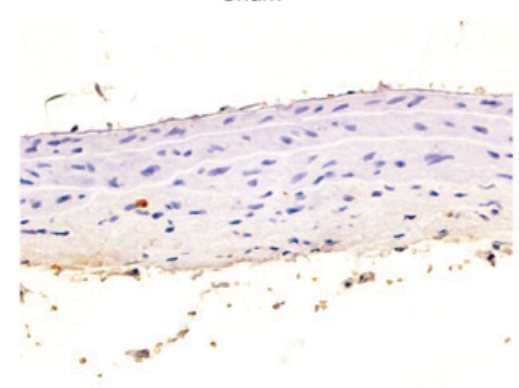

Vascular injury+LPA

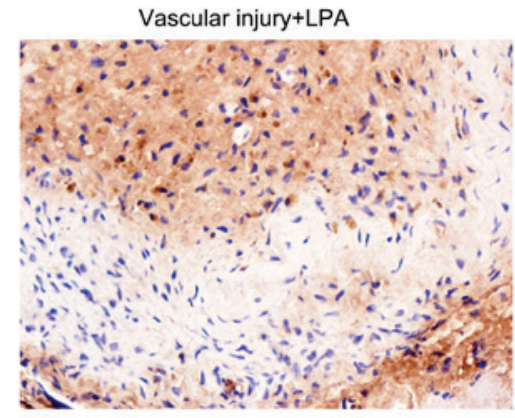

Vascular injury
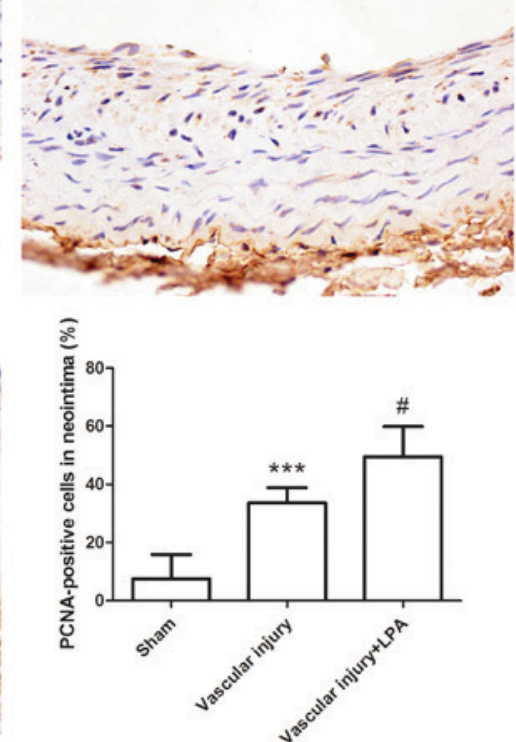

Figure 2. LPA enhances vascular injury-induced elevated PCNA expression. PCNA levels in the carotid artery tissues in each group were detected by immunohistochemistry. Magnification, $x 400$. The percentage of PCNA-positive cells in neointima was recorded. Data are presented as the means \pm standard deviation. Typical images are presented ( $\mathrm{n}=6 /$ group). ${ }^{* * *} \mathrm{P}<0.001$ compared with the sham group; ${ }^{\#} \mathrm{P}<0.05$ compared with the vascular injury group. LPA, lysophosphatidic acid; PCNA, proliferating cell nuclear antigen. 

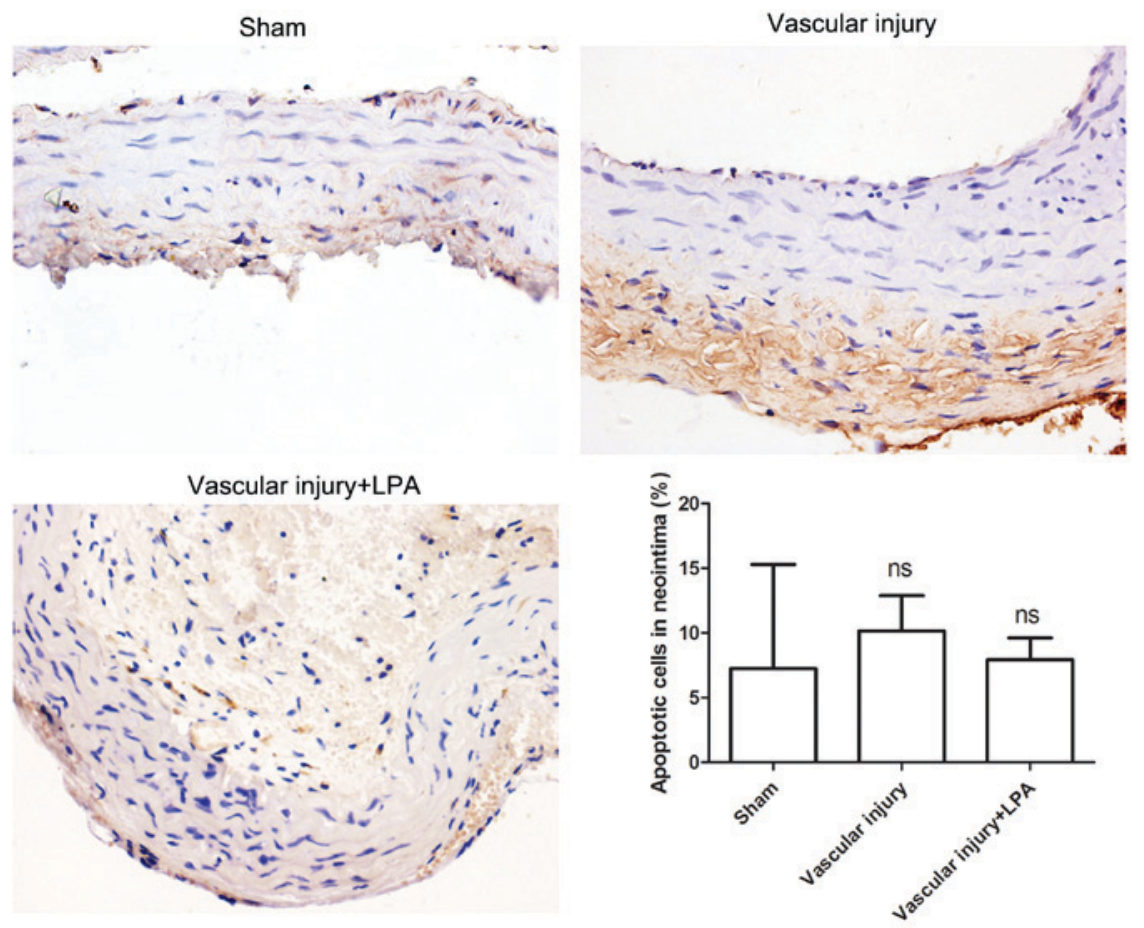

Figure 3. LPA exerts no effect on apoptosis in neointima. Apoptosis of carotid artery tissues was detected by TUNEL assay. Magnification, x400. The percentage of TUNEL-positive cells in neointima was recorded. Data are presented as the means \pm standard deviation. Typical images are presented ( $\mathrm{n}=6$ animals/group). LPA, lysophosphatidic acid; ns, not significant; TUNEL, terminal deoxynucleotidyl transferase-mediated dUTP nick end labeling.

of apoptosis in carotid artery tissues was investigated. The results of the TUNEL assay demonstrated that there was no significant difference in apoptotic levels among the various groups (Fig. 3). In addition, the protein expression levels of caspase-3, Bax and Bcl-2 were detected to evaluate apoptosis in each group. The results revealed that there was no significant difference in the expression levels of caspase-3 among the groups (Fig. 4A). In the vascular injury group, the expression levels of Bax were significantly increased compared with the sham group (Fig. 4B). In addition, the expression levels of $\mathrm{Bcl}-2$ were significantly increased in the vascular injury group compared with the sham group (Fig. 4C). However, the Bax/Bcl-2 ratio exhibited no significant difference between the vascular injury and sham groups (Fig. 4D). Furthermore, the expression levels of Bax and Bcl-2 revealed no significant difference between the vascular injury and vascular injury + LPA groups (Fig. 4B-D). These results were consistent with the results of TUNEL assay, and demonstrated that LPA had no effect on apoptosis in vascular injury-induced neointima.

The levels of autophagy were elevated in response to vascular injury, as determined by the expression levels of LC3 II/I and p62 via western blotting. The results demonstrated that, in the vascular injury group, the expression levels of LC3 II/I and p62 were significantly increased and decreased, respectively, compared with in the sham group, thus indicating activation of autophagy. However, upon treatment with LPA, the expression levels of LC3 II/I were significantly decreased and those of p62 were significantly increased compared with the vascular injury group (Fig. 5). These results demonstrated that activation of autophagy induced by vascular injury may be inhibited by LPA.
LPA affects inflammation and oxidative stress induced by vascular injury. In the present study, the expression levels of TNF- $\alpha$, IL-1 $\beta$ and IL-10 were measured, in order to evaluate inflammatory status. In the vascular injury group, the expression levels of TNF- $\alpha$, IL-1 $\beta$ and IL-10 were significantly increased compared with the sham group (Fig. 6). In the vascular injury + LPA group, the expression levels of TNF- $\alpha$ and IL-1 $\beta$ were significantly enhanced upon treatment with LPA compared with the vascular injury group (Fig. 6A and B); however, the expression levels of IL-10 in the vascular injury + LPA group exhibited a slight, but not significant decrease following treatment with LPA (Fig. 6C). These results suggested that LPA may affect vascular injury-induced inflammation.

Since oxidative stress also contributes to formation of neointima, the levels of MDA, MPO and SOD were measured using corresponding kits. The results indicated that, compared with the sham group, the MDA and MPO levels in the vascular injury group were significantly increased (Fig. 7A and B); conversely, the expression levels of SOD were significantly decreased in the vascular injury group (Fig. 7C). In addition, in the vascular injury + LPA group, the increased MDA and MPO levels caused by vascular injury were significantly enhanced by LPA treatment, whereas SOD levels were decreased; however, this was not significant (Fig. 7). These results indicated that LPA may enhance vascular injury-induced oxidative stress.

\section{Discussion}

LPA serves an important role in the cardiocerebral vascular system. In the present study, the effects of LPA on neointimal formation following vascular injury were investigated. The 
A

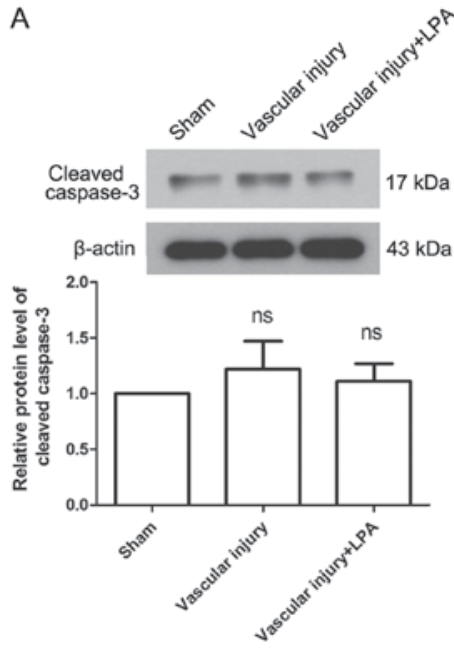

C

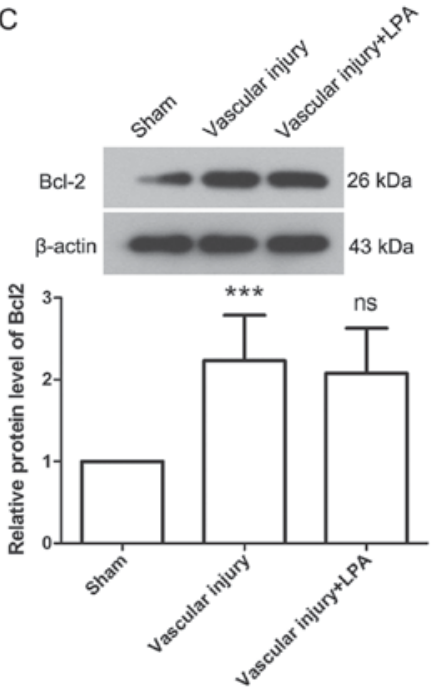

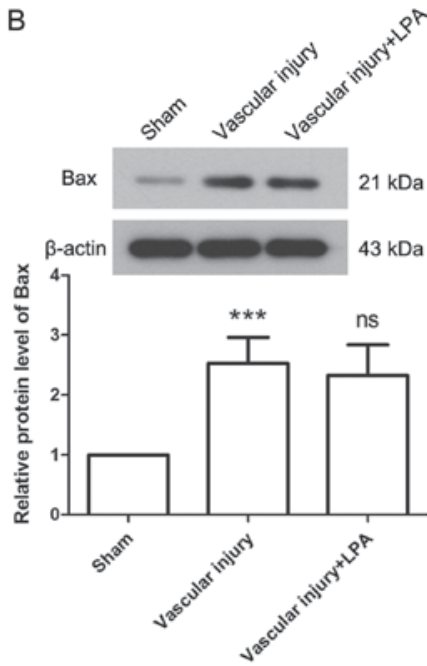

D

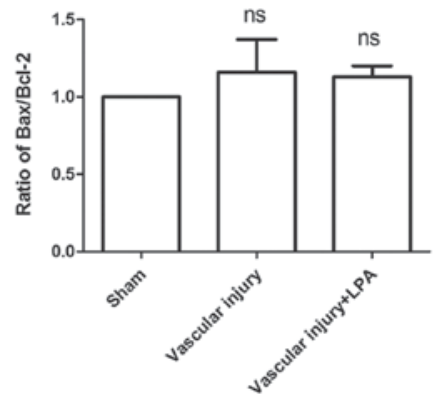

Figure 4. LPA exerts no effect on apoptosis in neointima. Protein expression levels of (A) cleaved caspase-3, (B) Bax and (C) Bcl-2 were detected by western blotting. $\beta$-actin was used as an internal reference. (D) Bax/Bcl-2 ratio. Data are presented as the means \pm standard deviation (n=6 animals/group). ${ }^{* * *} \mathrm{P}<0.001$ compared with the sham group. Bax, Bcl-2-associated X; Bcl-2, B-cell lymphoma-2; LPA, lysophosphatidic acid; ns, not significant.
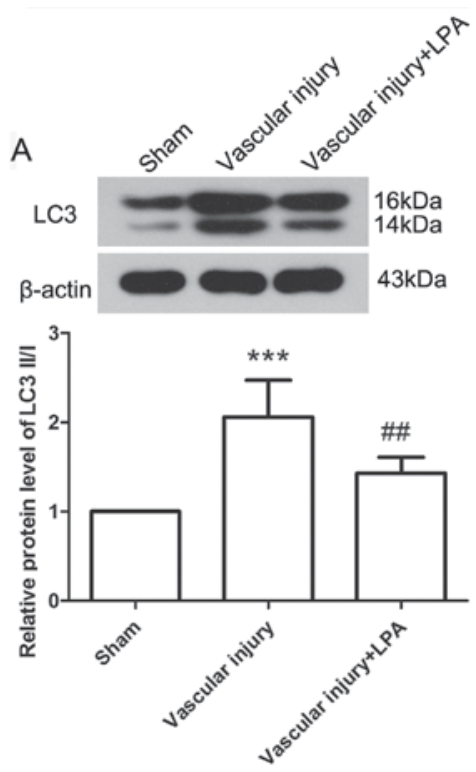
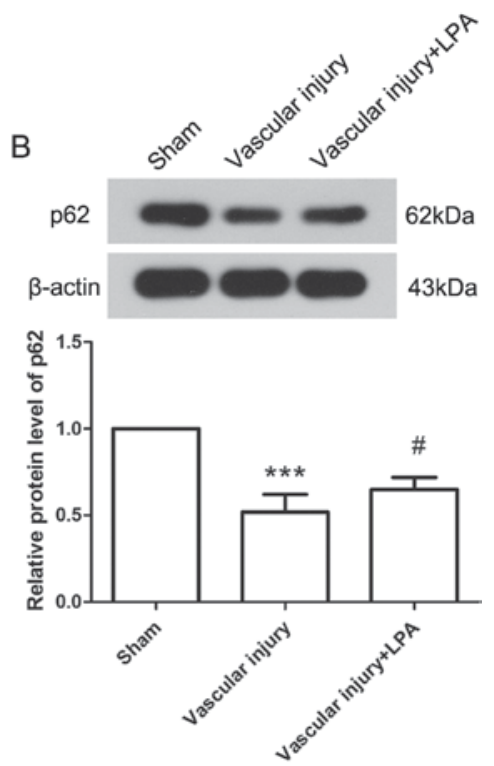

Figure 5. LPA modulates autophagy within injured carotid arteries. (A) Expression levels of LC3 II/I in each group were detected by western blotting. $\beta$-actin served as an internal reference. (B) Western blotting was performed to detect the expression levels of p62 in each group. Data are presented as the means \pm standard deviation ( $\mathrm{n}=6$ animals/group). ${ }^{* * * *} \mathrm{P}<0.001$ compared with the sham group; ${ }^{*} \mathrm{P}<0.05$ and ${ }^{\# *} \mathrm{P}<0.01$ compared with the vascular injury group. LC3 II/I, microtubule-associated protein 1A/1B light chain 3; LPA, lysophosphatidic acid. 

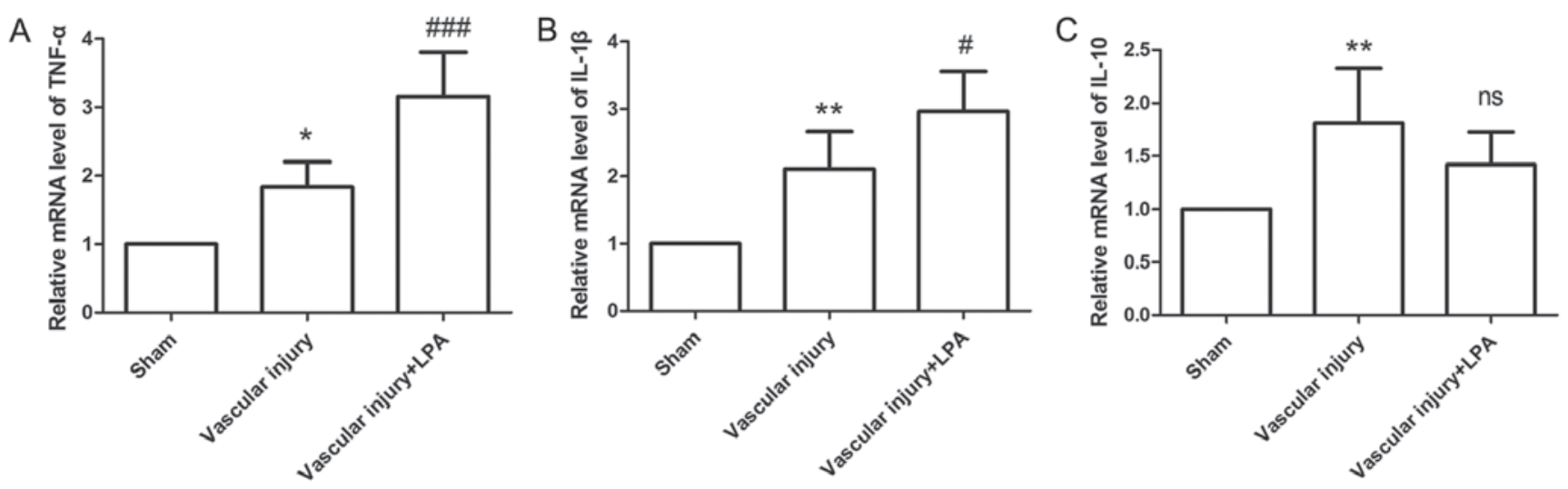

Figure 6. LPA affects inflammation within injured carotid arteries. The expression levels of (A) TNF- $\alpha$, (B) IL-1 $\beta$ and (C) IL-10 in the carotid artery tissues of each group were detected by reverse transcription-quantitative polymerase chain reaction. The relative mRNA expression levels were calculated using the $2^{-\Delta \Delta C q}$ method. Data are presented as the means \pm standard deviation ( $\mathrm{n}=6$ animals/group). ${ }^{*} \mathrm{P}<0.05$ and ${ }^{* * *} \mathrm{P}<0.01$ compared with the sham group; ${ }^{\#} \mathrm{P}<0.05$ and \#\#\# $\mathrm{P}<0.001$ compared with the vascular injury group. IL, interleukin; LPA, lysophosphatidic acid; ns, not significant; TNF, tumor necrosis factor.

A

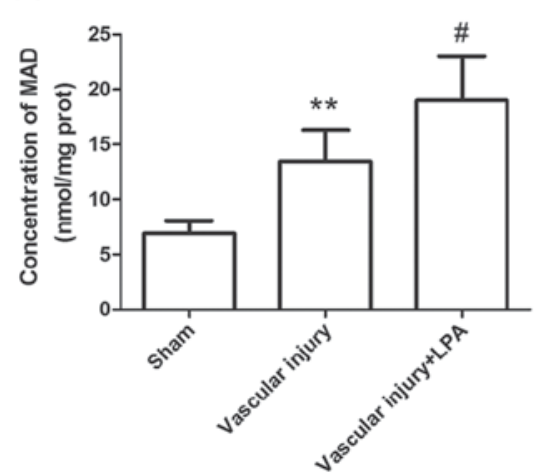

B

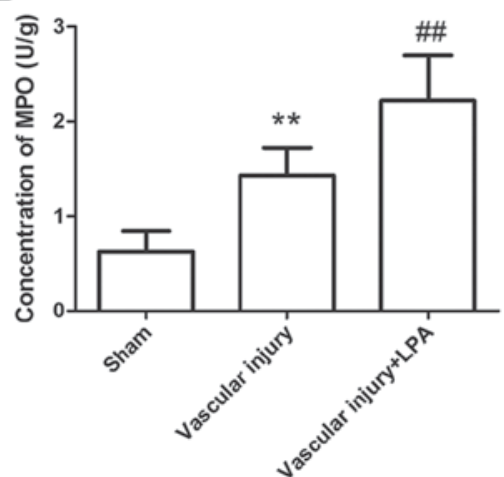

C

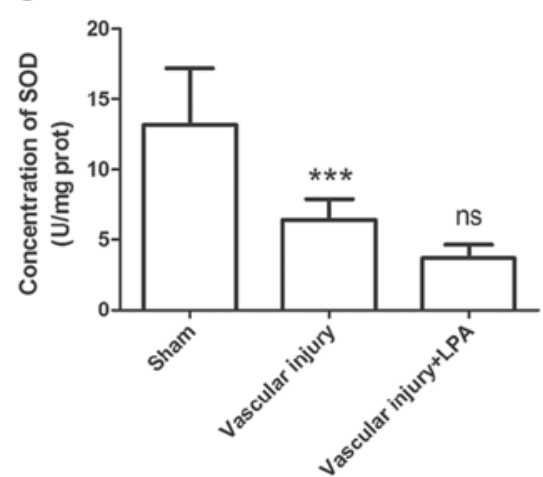

Figure 7. LPA affects oxidative stress in injured carotid arteries. The levels of (A) MDA, (B) MPO and (C) SOD in the carotid artery tissues of each group were measured with corresponding kits. Data are presented as the means \pm standard deviation $\left(\mathrm{n}=6\right.$ animals/group). ${ }^{* *} \mathrm{P}<0.01$ and ${ }^{* * * *} \mathrm{P}<0.001$ compared with the sham group; ${ }^{\#} \mathrm{P}<0.05$ and ${ }^{\# \#} \mathrm{P}<0.01$ compared with the vascular injury group. LPA, lysophosphatidic acid; MDA, malondialdehyde; MPO, myeloperoxidase; ns, not significant; SOD, superoxide dismutase.

results of the present study revealed that LPA enhanced neointimal hyperplasia in the injured carotid arteries by modulating proliferation, autophagy, inflammation and oxidative stress. Conversely, LPA exerted no significant effects on apoptosis in injured carotid arteries. These results suggested that LPA may contribute to the pathogenesis of atherosclerosis.

The expression levels of LPA are elevated in atherosclerotic lesions $(18,19)$, and LPA can induce rapid activation of platelets, stimulate angiogenesis and regulate the expression of vascular endothelial growth factor (19-21). The present study revealed that LPA enhanced vascular injury-induced neointimal hyperplasia via the modulation of proliferation, autophagy, inflammation and oxidative stress. In concordance with the present study, Zhang et al (3) suggested that LPA may induce neointimal formation in a rat carotid artery model by activating peroxisome proliferator-activated-receptor $\gamma$. Subramanian et al (13) also reported that treatment with Ki16425, which blocks LPA receptors (LPA1 and LPA3), inhibits neointimal formation. These findings indicated that LPA may contribute to the pathological process of atherosclerosis. In addition, Kritikou et al (14) suggested that the inhibition of LPA receptors may reduce the size of atherosclerotic plaques. Since LPA serves such a critical role in atherosclerosis, it may be considered a promising therapeutic target for atherosclerosis.

LPA can induce DNA replication and mitosis (22,23); excessive cell proliferation in arterial walls contributes to the growth of plaques (5). Vascular smooth muscle cells are the dominant type of cells that contribute to atherosclerotic lesions. The proliferation and migration of vascular smooth muscle cells contribute to the pathogenesis of atherosclerosis (24). LPA has previously been reported to serve as a mitogenic growth factor of vascular smooth muscle cells, thus promoting their proliferation and affecting their migration $(22,25,26)$. LPA also induces the proliferation and migration of vascular endothelial cells, and activates endothelial cells to produce adhesion molecules and secrete inflammatory cytokines, which also contribute to the pathogenesis of atherosclerosis (27-29). Furthermore, LPA affects the migration of fibroblasts and monocytes (30), which are important for neointimal formation and the pathogenesis of atherosclerosis (31). In the present study, the injured carotid artery tissues exhibited higher PCNA expression levels 
following LPA treatment, indicating enhanced proliferation. Therefore, LPA may contribute to the pathogenesis of atherosclerosis.

LPA serves an indefinite role in cell apoptosis. LPA has been reported to exert no effect on the apoptosis of colon cancer cells, but may increase their proliferation (32). Conversely, LPA has been indicated to induce apoptosis, but protect against cisplatin-induced apoptosis in cervical cancer cells $(33,34)$. The effects of LPA have been reported to promote epithelial cell apoptosis following lung injury, and promote the resistance of lung fibroblasts to apoptosis (35). In the present study, LPA enhanced neointimal hyperplasia caused by vascular injury, but exerted no effect on the apoptosis of vascular cells, as evidenced by TUNEL assay and unaltered caspase-3 levels and Bax/Bcl-2 ratios. LPA also protects macrophages from apoptosis, promoting atherosclerotic lesion formation (36).

Autophagy, which is activated by stress, nutrient deprivation and toxic agents, is a conserved process that degrades long-lived proteins, damaged organelles and macromolecular aggregates for recycling (37). Autophagy serves an important role in cholesterol metabolism and contributes to the pathological processes of atherosclerosis (38); however, the role of autophagy in atherosclerosis is complex, with both detrimental and protective effects (39). Ye et al (40) reported that, in injured carotid arteries, activation of autophagy influx appears in the neointima; consistently, in the present study, autophagy was activated in injured carotid arteries. Notably, the activation of autophagy in injured arteries was eliminated upon treatment with LPA in the present study. Grootaert et al (41) demonstrated that defective autophagy may promote post-injury neointimal formation. Therefore, LPA-induced suppression of autophagy may contribute to enhanced neointimal hyperplasia.

Atherosclerosis is associated with chronic inflammation. In the present study, LPA was reported to enhance the elevated expression levels of inflammatory cytokines, TNF- $\alpha$ and IL-1 $\beta$, in injured carotid arteries. These results revealed that LPA may enhance vascular injury-induced inflammation. Consistent with the findings of the present study, previous studies demonstrated that LPA may induce the expression of IL-1 $\beta$ in macrophages, thus contributing to the development of atherosclerosis $(42,43)$. It has also been reported that LPA promotes the synthesis and release of TNF- $\alpha$ by T lymphocytes (44). IL-10 is a well-known anti-inflammatory cytokine, which serves a protective role against atherosclerosis (45-47). Notably, the expression levels of IL-10 are increased in advanced or unstable atherosclerotic plaques $(48,49)$. In the present study, the expression levels of IL-10 in injured carotid arteries were elevated post-vascular injury; however, LPA exerted no effect on the elevated expression levels of IL-10. The recruitment of immune cells also contributes to the pathology of atherosclerosis. LPA has been revealed to stimulate the accumulation of macrophages, promote the migration and adhesion of monocytes to endothelium, and contribute to the aggravation of atherosclerotic plaques (50-52).

Atherosclerosis is also associated with oxidative stress-induced conditions (39). In early atherosclerotic lesions, oxidative stress is activated, thus resulting in oxidative modification of low-density lipoprotein, which is a pathogenic factor for atherosclerosis (53). The present study revealed that oxidative stress induced by vascular injury was enhanced upon treatment with LPA, thus indicating that enhanced oxidative stress may be associated with the neointimal-promoting effects of LPA. According to the literature, oxidative stress is also involved in the endothelial cytotoxicity of LPA (54).

In conclusion, the present study demonstrated that LPA may enhance neointimal hyperplasia by modulating proliferation, autophagy, inflammation and oxidative stress, but not apoptosis, within injured carotid arteries. The findings of the present study indicated that LPA may contribute to the pathogenesis of atherosclerosis and may be considered a promising target for the treatment of atherosclerosis.

\section{Acknowledgements}

Not applicable.

\section{Funding}

No funding was received.

\section{Availability of data and materials}

All data generated or analyzed during this study are included in this published article.

\section{Authors' contributions}

XS conceptualized the study design and wrote the manuscript. $\mathrm{XS}, \mathrm{JZ}, \mathrm{FL}, \mathrm{TZ}$ and TG performed the experiments and analyzed the data.

\section{Ethics approval and consent to participate}

The present study was approved by the Ethics Committee of The People's Hospital of China Medical University (Liaoning, China).

\section{Consent for publication}

Not applicable.

\section{Competing interests}

The authors declare that they have no competing interests.

\section{References}

1. Weber $\mathrm{C}$ and Noels $\mathrm{H}$ : Atherosclerosis: Current pathogenesis and therapeutic options. Nat Med 17: 1410-1422, 2011.

2. Schober A and Siess W: Lysophosphatidic acid in atherosclerotic diseases. Br J Pharmacol 167: 465-482, 2012.

3. Zhang C,Baker DL, Yasuda S, Makarova N, Balazs L, Johnson LR, Marathe GK, McIntyre TM, Xu Y, Prestwich GD, et al: Lysophosphatidic acid induces neointima formation through PPARgamma activation. J Exp Med 199: 763-774, 2004.

4. Clowes AW, Clowes MM, Fingerle J and Reidy MA: Regulation of smooth muscle cell growth in injured artery. J Cardiovasc Pharmacol 14 (Suppl 6): S12-S15, 1989.

5. Fuster JJ, Fernandez P, Gonzalez-Navarro H, Silvestre C, Nabah YN and Andres V: Control of cell proliferation in atherosclerosis: Insights from animal models and human studies. Cardiovasc Res 86: 254-264, 2010.

6. Lusis AJ: Atherosclerosis. Nature 407: 233-241, 2000. 
7. Sheng X, Yung YC, Chen A and Chun J: Lysophosphatidic acid signalling in development. Development 142: 1390-1395, 2015.

8. Choi JW, Herr DR, Noguchi K, Yung YC, Lee CW, Mutoh T, Lin ME, Teo ST, Park KE, Mosley AN and Chun J: LPA receptors: Subtypes and biological actions. Annu Rev Pharmaco Toxicol 50: 157-186, 2010.

9. Lin ME,Herr DR and Chun J: Lysophosphatidic acid (LPA) receptors: Signaling properties and disease relevance. Prostaglandins Other Lipid Mediat 91: 130-138, 2010

10. Smyth SS, Cheng HY, Miriyala S, Panchatcharam M and Morris AJ: Roles of lysophosphatidic acid in cardiovascular physiology and disease. Biochim Biophys Acta 1781: 563-570, 2008.

11. Siess W and Tigyi G: Thrombogenic and atherogenic activities of lysophosphatidic acid. J Cell Biochem 92: 1086-1094, 2004.

12. Yoshida K, Nishida W, Hayashi K, Ohkawa Y, Ogawa A, Aoki J, Arai H and Sobue K: Vascular remodeling induced by naturally occurring unsaturated lysophosphatidic acid in vivo. Circulation 108: 1746-1752, 2003.

13. Subramanian P, Karshovska E, Reinhard P, Megens RT, Zhou Z, Akhtar S, Schumann U, Li X, van Zandvoort M, Ludin C, et al: Lysophosphatidic acid receptors LPA1 and LPA3 promote CXCL12-mediated smooth muscle progenitor cell recruitment in neointima formation. Circ Res 107: 96-105, 2010.

14. Kritikou E, van Puijvelde GH, van der Heijden T, van Santbrink PJ, Swart M, Schaftenaar FH, Kröner MJ, Kuiper J and Bot I: Inhibition of lysophosphatidic acid receptors 1 and 3 attenuates atherosclerosis development in LDL-receptor deficient mice. Sci Rep 6: 37585, 2016.

15. Research NRCUIfLA: Guide for the care and use of laboratory animals. Washington (DC), National Academies Press (US), 1996.

16. Livak KJ and Schmittgen TD: Analysis of relative gene expression data using real-time quantitative PCR and the 2(-Delta Delta C(T)) method. Methods 25: 402-408, 2001.

17. Yu H, Clarke MC, Figg N, Littlewood TD and Bennett MR Smooth muscle cell apoptosis promotes vessel remodeling and repair via activation of cell migration, proliferation and collagen synthesis. Arterioscler Thromb Vasc Biol 31: 2402-2409, 2011.

18. Bot M, Bot I, Lopez-Vales R, van de Lest CH, Saulnier-Blache JS Helms JB, David S, van Berkel TJ and Biessen EA: Atherosclerotic lesion progression changes lysophosphatidic acid homeostasis to favor its accumulation. Am J Pathol 176: 3073-3084, 2010.

19. Rother E, Brandl R, Baker DL, Goyal P, Gebhard H, Tigyi G and Siess W: Subtype-selective antagonists of lysophosphatidic Acid receptors inhibit platelet activation triggered by the lipid core of atherosclerotic plaques. Circulation 108: 741-747, 2003.

20. Sweat RS, Azimi MS, Suarez-Martinez AD, Katakam P and Murfee WL: Lysophosphatidic acid does not cause blood/lymphatic vessel plasticity in the rat mesentery culture model. Physiol Rep 4: e12857, 2016.

21. Rivera-Lopez CM, Tucker AL and Lynch KR: Lysophosphatidic acid (LPA) and angiogenesis. Angiogenesis 11: 301-310, 2008.

22. Kim J, Keys JR and Eckhart AD: Vascular smooth muscle migration and proliferation in response to lysophosphatidic acid (LPA) is mediated by LPA receptors coupling to Gq. Cell Signal 18: $1695-1701,2006$

23. Xu YJ, Rathi SS, Chapman DC, Arneja AS and Dhalla NS: Mechanisms of lysophosphatidic acid-induced DNA synthesis in vascular smooth muscle cells. J Cardiovasc Pharmacol 41: 381-387, 2003

24. Chappell J, Harman JL, Narasimhan VM, Yu H, Foote K, Simons BD, Bennett MR and Jørgensen HF: Extensive proliferation of a subset of differentiated, yet plastic, medial vascular smooth muscle cells contributes to neointimal formation in mouse injury and atherosclerosis models. Circ Res 119: 1313-1323, 2016

25. Komachi M, Damirin A, Malchinkhuu E, Mogi C, Tobo M, Ohta H, Sato K, Tomura H and Okajima F: Signaling pathways involved in DNA synthesis and migration in response to lysophosphatidic acid and low-density lipoprotein in coronary artery smooth muscle cells. Vascul Pharmacol 50: 178-184, 2009.

26. Teo ST, Yung YC, Herr DR and Chun J: Lysophosphatidic acid in vascular development and disease. IUBMB Life 61: 791-799, 2009.

27. Lee H, Goetzl EJ and An S: Lysophosphatidic acid and sphingosine 1-phosphate stimulate endothelial cell wound healing. Am J Physiol Cell Physiol 278: C612-C618, 2000.

28. Lee H, Lin CI, Liao JJ, Lee YW, Yang HY, Lee CY, Hsu HY and Wu HL: Lysophospholipids increase ICAM-1 expression in HUVEC through a Gi- and NF-kappaB-dependent mechanism. Am J Physiol Cell Physiol 287: C1657-C1666, 2004.
29. Lin CI, Chen CN, Chen JH and Lee H: Lysophospholipids increase IL- 8 and MCP-1 expressions in human umbilical cord vein endothelial cells through an IL-1-dependent mechanism. J Cell Biochem 99: 1216-1232, 2006.

30. Pilquil C, Dewald J, Cherney A, Gorshkova I, Tigyi G, English D, Natarajan V and Brindley DN: Lipid phosphate phosphatase-1 regulates lysophosphatidate-induced fibroblast migration by controlling phospholipase D2-dependent phosphatidate generation. J Biol Chem 281: 38418-38429, 2006.

31. Sartore S, Chiavegato A, Faggin E, Franch R, Puato M, Ausoni S and Pauletto P: Contribution of adventitial fibroblasts to neointima formation and vascular remodeling: From innocent bystander to active participant. Circ Res 89: 1111-1121, 2001.

32. Leve F, Peres-Moreira RJ, Binato R, Abdelhay E and Morgado-Diaz JA: LPA induces colon cancer cell proliferation through a cooperation between the ROCK and STAT-3 pathways. PLoS One 10: e0139094, 2015

33. Dong Y, Wu Y, Cui MZ and Xu X: Lysophosphatidic acid triggers apoptosis in HeLa cells through the upregulation of tumor necrosis factor receptor superfamily member 21 . Mediators Inflamm 2017: 2754756, 2017.

34. Sui Y, Yang Y, Wang J, Li Y, Ma H, Cai H, Liu X, Zhang Y, Wang S, Li Z, et al: Lysophosphatidic acid inhibits apoptosis induced by cisplatin in cervical cancer cells. Biomed Res Int 2015: 598386, 2015.

35. Funke M, Zhao Z, Xu Y, Chun J and Tager AM: The lysophosphatidic acid receptor LPA1 promotes epithelial cell apoptosis after lung injury. Am J Respir Cell Mol Biol 46: 355-364, 2012

36. Koh JS, Lieberthal W, Heydrick S and Levine JS Lysophosphatidic acid is a major serum noncytokine survival factor for murine macrophages which acts via the phosphatidylinositol 3-kinase signaling pathway. J Clin Invest 102: 716-727, 1998

37. Rajawat YS and Bossis I: Autophagy in aging and in neurodegenerative disorders. Hormones (Athens) 7: 46-61, 2008

38. Ouimet M, Ediriweera H, Afonso MS, Ramkhelawon B, Singaravelu R, Liao X, Bandler RC, Rahman K, Fisher EA, Rayner KJ, et al: microRNA-33 regulates macrophage autophagy in atherosclerosis. Arterioscler Thromb Vasc Biol 37: 1058-1067, 2017.

39. Perrotta I and Aquila S: The role of oxidative stress and autophagy in atherosclerosis. Oxid Med Cell Longev 2015: 130315, 2015

40. Ye LX, Yu J, Liang YX, Zeng JS, Huang RX and Liao SJ: Beclin 1 knockdown retards re-endothelialization and exacerbates neointimal formation via a crosstalk between autophagy and apoptosis. Atherosclerosis 237: 146-154, 2014.

41. Grootaert MO, da Costa Martins PA, Bitsch N, Pintelon I, De Meyer GR, Martinet W and Schrijvers DM: Defective autophagy in vascular smooth muscle cells accelerates senescence and promotes neointima formation and atherogenesis. Autophagy 11: 2014-2032, 2015

42. Kirii H, Niwa T, Yamada Y, Wada H, Saito K, Iwakura Y, Asano M, Moriwaki H and Seishima M: Lack of interleukin-lbeta decreases the severity of atherosclerosis in ApoE-deficient mice. Arterioscler Thromb Vasc Biol 23: 656-660, 2003.

43. Chang CL, Lin ME, Hsu HY, Yao CL, Hwang SM, Pan CY, Hsu CY and Lee H: Lysophosphatidic acid-induced interleukin-1 beta expression is mediated through $\mathrm{Gi} / \mathrm{Rho}$ and the generation of reactive oxygen species in macrophages. J Biomed Sci 15 357-363, 2008

44. Zhang D, Zhang Y, Zhao C, Zhang W, Shao G and Zhang H: Effect of lysophosphatidic acid on the immune inflammatory response and the connexin 43 protein in myocardial infarction. Exp Ther Med 11: 1617-1624, 2016.

45. Pinderski Oslund LJ, Hedrick CC, Olvera T, Hagenbaugh A, Territo M, Berliner JA and Fyfe AI: Interleukin-10 blocks atherosclerotic events in vitro and in vivo. Arterioscler Thromb Vasc Biol 19: 2847-2853, 1999.

46. Pinderski LJ, Fischbein MP, Subbanagounder G, Fishbein MC, Kubo N, Cheroutre H, Curtiss LK, Berliner JA and Boisvert WA: Overexpression of interleukin-10 by activated T lymphocytes inhibits atherosclerosis in LDL receptor-deficient Mice by altering lymphocyte and macrophage phenotypes. Circ Res 90 1064-1071, 2002

47. Mallat Z, Besnard S, Duriez M, Deleuze V, Emmanuel F, Bureau MF, Soubrier F, Esposito B, Duez H, Fievet C, et al: Protective role of interleukin-10 in atherosclerosis. Circ Res 85: e17-e24, 1999. 
48. Mallat Z, Heymes C, Ohan J, Faggin E, Leseche G and Tedgui A Expression of interleukin-10 in advanced human atherosclerotic plaques: Relation to inducible nitric oxide synthase expression and cell death. Arterioscler Thromb Vasc Biol 19: 611-616, 1999.

49. Nishihira K, Imamura T, Yamashita A, Hatakeyama K, Shibata Y, Nagatomo Y, Date H, Kita T, Eto T and Asada Y: Increased expression of interleukin-10 in unstable plaque obtained by directional coronary atherectomy. Eur Heart J 27: 1685-1689, 2006.

50. Zhou Z, Subramanian P, Sevilmis G, Globke B, Soehnlein O, Karshovska E, Megens R, Heyll K, Chun J, Saulnier-Blache JS, et al: Lipoprotein-derived lysophosphatidic acid promotes atherosclerosis by releasing CXCL1 from the endothelium. Cell Metab 13: 592-600, 2011.

51. Gustin C, Van Steenbrugge $M$ and Raes M: LPA modulates monocyte migration directly and via LPA-stimulated endothelial cells. Am J Physiol Cell Physiol 295: C905-C914, 2008.
52. Bot M, de Jager SC, MacAleese L, Lagraauw HM, van Berkel TJ, Quax PH, Kuiper J, Heeren RM, Biessen EA and Bot I: Lysophosphatidic acid triggers mast cell-driven atherosclerotic plaque destabilization by increasing vascular inflammation. J Lipid Res 54: 1265-1274, 2013.

53. Sparrow CP and Olszewski J: Cellular oxidative modification of low density lipoprotein does not require lipoxygenases. Proc Natl Acad Sci USA 89: 128-131, 1992.

54. Brault S, Gobeil F Jr, Fortier A, Honore JC, Joyal JS, Sapieha PS, Kooli A, Martin E, Hardy P, Ribeiro-da-Silva A, et al: Lysophosphatidic acid induces endothelial cell death by modulating the redox environment. Am J Physiol Regul Integr Comp Physiol 292: R1174-R1183, 2007.

(i) $\ominus$ This work is licensed under a Creative Commons Attribution-NonCommercial-NoDerivatives 4.0 International (CC BY-NC-ND 4.0) License. 